Radiologe 2019·59:859

https://doi.org/10.1007/s00117-019-00596-y

(C) Springer Medizin Verlag GmbH, ein Teil von Springer Nature 2019

\section{S. Trattnig' ' C. Herold ${ }^{2}$}

'Exzellenzzentrum für Hochfeld MR, Universitätsklinik für Radiologie und Nuklearmedizin, Medizinische Universität Wien, Wien, Österreich

${ }^{2}$ Universitätsklinik für Radiologie und Nuklearmedizin, Medizinische Universität Wien, Wien, Österreich

\title{
Magnetresonanztomographische Sicherheit von (elektronischen) Implantaten und Devices
}

Liebe Leserinnen und Leser,

mit der zunehmenden Lebenserwartung und verbesserten Therapiemöglichkeiten der Medizin in den letzten 20 Jahren hat auch die Zahl an aktiven und passiven Implantaten, die therapeutisch am Patienten eingesetzt werden, deutlich zugenommen. Zudem hat sich die Magnetresonanztomographie (MRT) als diagnostische Modalität aufgrund ihres hohen Weichteilkontrastes und ihrer funktionellen Untersuchungsmöglichkeiten in vielen Bereichen als Methode der Wahl etabliert. Dies hat zur Folge, dass zunehmend mehr Patienten mit Implantaten im Laufe ihres Lebens eine MRT-Untersuchung benötigen.

Eine MRT bei einem Patienten mit einem aktiven oder passiven Implantat sicher durchzuführen, erfordert ein grundsätzliches Verständnis der MRWechselwirkungen mit magnetischem und elektrisch leitendem Material. Besondere Aufmerksamkeit erfordern aktive Implantate, denen zwei ausführliche Beiträge über die Aspekte der MR-Sicherheit gewidmet sind. Im Speziellen wird auf die sichere MRT-Untersuchung bei Patienten mit Herzschrittmachern und intrakardialen Defibrillatoren eingegangen. Letztere sind insofern von großer Bedeutung, als noch vor wenigen Jahren implantierte Herzschrittmacher als Kontraindikation für eine MRT-Untersuchung galten. Seit einigen Jahren werden nun fast ausschließlich bedingt sichere Herzschrittmacher entwickelt, die unter Einhaltung bestimmter Rahmenbedingungen eine sichere MRT-Untersu- chung ermöglichen. Deren Management im Zusammenspiel mit Kardiologie und Radiologie werden in diesem Heft ausführlich erörtert.

Neben den aktiven Implantaten sind auch passive Implantate hinsichtlich ihrer MR-Sicherheit zu überprüfen. Dazu sind eine Reihe von Testverfahren vorgesehen, auf welche ausführlich eingegangen wird. Schließlich wird dem Management in der Praxis ein besonderes Augenmerk gewidmet, insbesondere was die Implantat-Abklärung betrifft. Tipps und Guidelines sollen helfen, diese Abklärung auch im klinischen Routinebetrieb effizient durchführen zu können.

Seit 2017 ist das erste UltrahochfeldMRT-System mit einer Feldstärke von 7T von der US-amerikanischen Arzneimittelbehörde (FDA) zugelassen und auch CE-zertifiziert - somit steht nun auch die Ultrahochfeld-MRT für den vermehrten klinischen Einsatz zur Verfügung. Gerade bei der Hochfeld- ( 3 T) und insbesondere Ultrahochfeld-MRT (7T) sind noch höhere Ansprüche an die MR-Sicherheit zu stellen, da z. B. die spezifische Absorptionsrate (als Maß für die vom Körper aufgenommene Energie, die von den Spulen ausgesandt wird) nicht nur linear, sondern mit dem Quadrat der Feldstärke zunimmt - mit den damit verbundenen Risiken der Erwärmung bis hin zur Erhitzung und Verbrennung. Diese besondere Herausforderung höherer Feldstärken an die MRT-Sicherheit wird in einem Beitrag beleuchtet und entsprechende Verhaltensregeln in der Abklärung und Durchführung einer Hochfeld- und Ultrahochfeld-MRT bei Patienten mit Implantaten diskutiert.

Zusammenfassend hoffen die Herausgeber, mit diesem Themenheft einen Beitrag zur Verbesserung der MR-Sicherheit von Implantaten in der klinischen Anwendung leisten zu können.

Prof. Dr. Siegfried Trattnig

Prof. Dr. Christian Herold

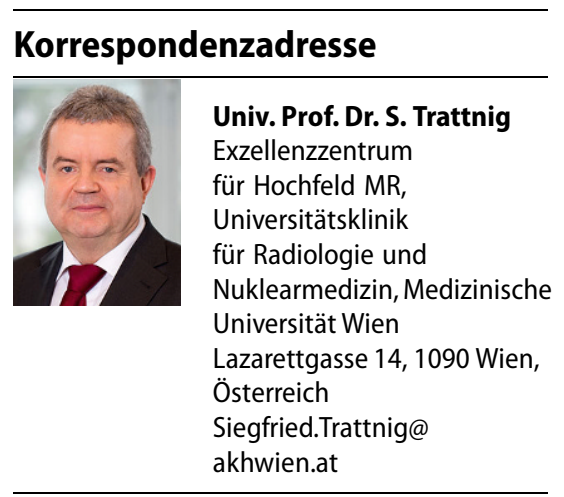

Interessenkonflikt. S. Trattnig gibt an, dass kein Interessenkonflikt besteht. 